\title{
Interplay between cigarette smoking and pulmonary reverse lipid transport
}

\author{
Éric Jubinville ${ }^{1,2}$, Maude Talbot ${ }^{1,2}$, Jean-Christophe Bérubé ${ }^{1,2}$, \\ Mélanie Hamel-Auger ${ }^{1,2}$, Michaël Maranda-Robitaille ${ }^{2}$, Marie-Josée Beaulieu², \\ Sophie Aubin ${ }^{2}$, Marie-Ève Paré ${ }^{2}$, David G. Kallend ${ }^{3}$, Benoit Arsenault ${ }^{2,4}$, \\ Yohan Bossé ${ }^{2,5}$ and Mathieu C. Morissette 2,4
}

\begin{abstract}
Affiliations: ${ }^{1}$ Faculty of Medicine, Université Laval, Quebec City, QC, Canada. ${ }^{2}$ Quebec Heart and Lung Institute - Université Laval, Quebec City, QC, Canada. ${ }^{3}$ The Medicines Company (Schweiz) GmbH, Zürich, Switzerland. ${ }^{4}$ Dept of Medicine, Université Laval, Quebec City, QC, Canada. ${ }^{5}$ Dept of Molecular Medicine, Université Laval, Quebec City, QC, Canada.
\end{abstract}

Correspondence: Mathieu C Morissette, 2725 Chemin Sainte-Foy, Québec, QC, Canada G1V 4 G5.

E-mail: mathieu.morissettedcriucpq.ulaval.ca

@ERSpublications

Smoking affects reverse lipid export mechanisms, represent a new pathological mechanism and therapeutic target http://ow.ly/g8pw30dWU8U

Cite this article as: Jubinville É, Talbot $\mathrm{M}$, Bérubé $\mathrm{J}-\mathrm{C}$, et al. Interplay between cigarette smoking and pulmonary reverse lipid transport. Eur Respir J 2017; 50: 1700681 [https://doi.org/10.1183/ 13993003.00681-2017].

ABSTRACT Reverse lipid transport is critical to maintain homeostasis. Smoking causes lipid accumulation in macrophages, therefore suggesting suboptimal reverse lipid transport mechanisms. In this study, we investigated the interplay between smoking and reverse lipid transport and the consequences on smoking-induced lung and peripheral alterations.

To investigate the relationship between smoking and reverse lipid transport, we used a clinical lung gene expression dataset and a mouse model of cigarette smoke exposure. We also used ApoA-1 ${ }^{-/-}$mice, with reduced reverse lipid transport capacity, and a recombinant ApoA-1 Milano/phospholipid complex (MDCO-216) to boost reverse lipid transport. Cellular and functional analyses were performed on the lungs and impact on body composition was also assessed.

Smoking affects pulmonary expression of abcal, abcg1, apoe and scarb1 in both mice and humans, key genes involved in reverse lipid transport. In mice, the capacity of bronchoalveolar lavage fluid and serum to stimulate cholesterol efflux in macrophages was increased after a single exposure to cigarette smoke. ApoA $-1^{-1-}$ mice showed increased lung neutrophilia, larger macrophages and greater loss in lean mass in response to smoking, whereas treatment with MDCO-216 reduced the size of macrophages and increased the lean mass of mice exposed to cigarette smoke.

Altogether, this study shows a functional interaction between smoking and reverse lipid transport, and opens new avenues for better understanding the link between metabolic and pulmonary diseases related to smoking.

This article has supplementary material available from erj.ersjournals.com

Received: March 312017 | Accepted after revision: June 062017

Support statement: Operating research grants from the Flight Attendant Medical Research Institute (FAMRI), the National Sanitarium Association (NSA), and the Respiratory Health Network of the FRQS (RSR-FRQS) funded parts of this study. É. Jubinville and J-C. Bérubé are recipients of doctoral studentships from the Fonds de recherche Québec Santé (FRQS) (É. Jubinville and J-C. Bérubé) and the Canadian Respiratory Research Network (CRRN) (J-C. Bérubé only). B. Arsenault and M.C. Morissette are Junior Scholars of the FRQS. Y. Bossé holds a Canada Research Chair in Genomics of Heart and Lung Diseases. Funding information for this article has been deposited with the Crossref Funder Registry.

Conflict of interest: Disclosures can be found alongside this article at erj.ersjournals.com

Copyright OERS 2017 


\section{Introduction}

Reverse lipid transport first starts with the export of intracellular phospholipids and cholesterols to acceptors such as high-density lipoproteins (HDLs) present in the cellular environment [1]. HDLs then access the peripheral circulation, reaching the liver where they are capture and processed, and the lipid content repackaged or excreted. The archetype lipid transporters ABCA1 and ABCG1 are especially important in initiating this process by the exporting cell as they recognise and bind apolipoprotein A-1 (ApoA-1), the most abundant apolipoprotein in HDLs [2]. ABCA1 has special affinity for lipid-poor HDLs, such as pre- $\beta$-HDL, while ABCG1 interacts with more mature HDL molecules [2]. Reverse lipid transport therefore integrates in the great scheme of systemic lipid transport, preventing problematic lipid accumulation in peripheral tissues.

Reverse lipid transport is extremely important to pulmonary cells, especially alveolar macrophages and type II pneumocytes, as they process a significant load of lipids on a daily basis. At the alveolar level, most of these lipids originate from the pulmonary surfactant, a high-turnover vital structure composed of phospholipids $(\sim 85 \%)$, cholesterols $(\sim 10 \%)$ and proteins $(\sim 5 \%)$ that lowers alveolar surface tension [3]. Type II pneumocytes are responsible for pulmonary surfactant synthesis and recycling, while alveolar macrophages take care of its degradation when damaged [4]. In the lungs, deficiencies in reverse lipid transporters ATP-binding cassette A1 (ABCA1) and ABCG1 rapidly lead to lipid accumulation in alveolar macrophages and type II pneumocytes, as well as intra-alveolar pulmonary surfactant build-up, ultimately causing lung dysfunction [5-7]. Therefore, reverse lipid transport is a key function in the lung, and pulmonary surfactant and alveolar macrophage homeostasis.

Given the importance of reverse lipid transport in the lungs and the pathological consequences of its dysfunction, environmental exposures that damage lipid structures like the pulmonary surfactant may require increased surfactant degradation by macrophages and, as a consequence, acceleration of reverse lipid transport. Cigarette smoking has been well documented for causing pulmonary lipid damage [8-10]. In fact, upon exposure to cigarette smoke, alveolar macrophages become chronically enlarged, showing extensive intracellular lipid accumulation coupled with the release of numerous inflammatory mediators [11], suggesting suboptimal capabilities in reverse lipid transport. A recent pre-clinical study proposed that this foamy macrophage phenotype and early inflammatory response to cigarette smoke exposure is due, at least in part, to direct damage made to pulmonary lipids [11]. Similarly to pulmonary surfactant and cellular lipids, lipoproteins are exposed to biochemical damage by cigarette smoke. Lipoproteins damaged by cigarette smoke have compromised functionality, reducing their capacity to promote and support reverse lipid transport [12]. This could be relevant to chronic smoking-related lung disease, such as chronic obstructive pulmonary disease (COPD), where lung diffusion is reduced, small airways are dysfunctional, often leading to hyperinflation, and increased alveolar surface tension caused by compromised surfactant integrity could lead to excessive alveolar stretching and damage. Thus, we postulate that the progressive and constant pressure applied by cigarette smoke inhalation on pulmonary reverse lipid transport mechanisms may represent an underappreciated contributor to smoking-related lung pathologies, preventing macrophages from properly disposing of intracellular lipids and further promoting local inflammation. Moreover, improving the maintenance of pulmonary lipid homeostasis could have beneficial effects on lung functions but also on the peripheral manifestations of smoking, such as lean mass loss.

In this study, we first investigated the impact of cigarette smoke exposure on the levels of key genes involved in reverse lipid transport using clinical and pre-clinical samples and found that cigarette smoke exposure modulates the expression of the lipid transporters abcg1, abca1, and scarb1 as well as the apolipoprotein E (apoe) in the lungs of both humans and mice. Using our pre-clinical model of cigarette smoke exposure, we found that cigarette smoke exposure elicits a rapid increase in the ability of bronchoalveolar (BAL) fluid and serum to promote reverse lipid transport in macrophages. To investigate the impact of a dysfunctional reverse lipid transport capacity, mice lacking ApoA-1, the main apolipoprotein of HDLs, showed an exacerbated pulmonary and systemic response when exposed to cigarette smoke. Finally, administration of MDCO-216, a recombinant ApoA-1 Milano/phospholipid complex, was able to dampen the pulmonary and systemic effects of cigarette smoke exposure.

\section{Methods}

Human lung gene expression cohort and analyses

Messenger RNA levels of key genes involved in reverse lipid transport, namely abcg1, abcal, scarb1 and apoe, were investigated in a human lung gene expression dataset previously described [13]. Briefly, microarray data from 465 non-tumour lung specimens were obtained from patients that underwent lung cancer resection. Gene expression levels were adjusted for sex and age, and compared among never, former and current smokers. The elapsed time between smoking cessation and tissue collection was also 
considered among former smokers. Differences in gene expression levels were tested using a one-way ANOVA followed by a Tukey's multiple comparison tests.

Mice, cigarette smoke exposure and treatments

Female 6-8 weeks old BALB/c (Charles River, St-Constant, QC, Canada), C57BL/6 (Charles River), C57BL/6J (Jackson Laboratories, Bar Harbor, ME, USA) and ApoA-1-deficient (Jackson Laboratories) mice were exposed to the mainstream smoke of 3R4F research cigarettes (University of Kentucky, Lexington, KY, USA) for two consecutive hours per day, five days a week, for 2 or 8 weeks using the Promech SIU24 whole-body exposure system (Promech Lab AB, Vintrie, Sweden). MDCO-216 (20 or $100 \mathrm{mg} \cdot \mathrm{kg}^{-1}$ in $200 \mu \mathrm{L}$; kindly provided by The Medicines Company, Parsippany, NJ, USA), a recombinant ApoA-1 Milano/phospholipid complex, and saline were delivered intraperitoneally $2 \mathrm{~h}$ after every cigarette smoke exposure (2-week exposure protocol) or every Monday, Wednesday, and Friday during the last 4 weeks of exposure (8-week exposure protocol). Mice were housed according to the Canadian Council for Animal Care (CCAC) guidelines and Université Laval's animal research ethics board approved all procedures (animal utilisation protocol \#2014121-2).

\section{Lung harvesting and processing}

When lung function assessment was not required, mice were anesthetised with isoflurane. The lungs were removed from the chest cavity, the trachea was canulated, the right multi-lobed lung was attached with a suture string and the left lobe subjected to bronchoalveolar lavage (BAL), performing a first lavage with $250 \mu \mathrm{L}$ and a second with $200 \mu \mathrm{L}$ of cold PBS. The right lung was snap frozen in liquid nitrogen and kept at $-80^{\circ} \mathrm{C}$ for $\mathrm{qPCR}$ analyses. After $\mathrm{BAL}$, the left lobe was inflated with $10 \%$ formalin for paraffin embedding and tissue histology. BAL total cell concentration was determined using a haemacytometer. BAL was then centrifuged at $800 \mathrm{~g}$ for $10 \mathrm{~min}$ to pellet the cells and perform cytospins for cell differentials (Diff-Quik; Fisher Scientific, Ottawa, ON, Canada). At least 300 cells per cytospin were manually counted using the Image J software (v1.44o). The size of pulmonary macrophages was determined as previously described [11]. Thirty-five macrophages per cytospin per mouse were randomly picked and the surface area measured using the Image J Software. Lymphocytes (bright blue nucleus and small cytoplasm) were excluded from the analysis. The average size of the pulmonary macrophages from room air-exposed mice was set as $100 \%$. BAL fluid was collected in a separate tube and kept at $-80^{\circ} \mathrm{C}$.

\section{Human ApoA-1 ELISA}

Human ApoA-1 was assessed as a surrogate for MDCO-216 levels, a complex composed of recombinant human ApoA-1 Milano and phospholipids. Human ApoA-1 concentrations were assessed in mouse BAL fluid and serum samples using the human ApoA-1 ELISA kit (Abcam, Cambridge, UK) according to manufacturer's instructions.

\section{Quantitative PCR (qPCR)}

Lung lobes were homogenised in $1 \mathrm{~mL}$ of Trizol (Fisher Scientific) using PowerGen 125 polytron (Fisher Scientific). RNA was extracted according to manufacturer's instructions. RNA concentration and purity was determined using Take3 Trio Micro-Volume plate and Synergy H1 Hybrid Reader (BioTek, Winooski, VT, USA). RNA integrity was verified using agarose gel electrophoresis. Reverse transcription was performed on $1 \mu \mathrm{g}$ of RNA using the iScript ${ }^{\mathrm{TM}}$ Advanced cDNA Synthesis Kit for quantitative PCR (qPCR) (Bio-Rad, Mississauga, ON, Canada) according to manufacturer instructions. Abca1, abcg1, scarb1 and apoe lung mRNA expression was assessed by qPCR and normalised to hprt and rplp0 reference genes using the $\Delta \Delta \mathrm{Cq}$ method. All reactions were performed in duplicate or triplicate using SsoAdvanced ${ }^{\mathrm{TM}}$ Universal SYBR ${ }^{\infty}$ Green Supermix (Bio-Rad) and primers at $10 \mu \mathrm{M}$. The primers and qPCR conditions used for amplifications were optimised for each gene (abcal (NM_013454): Fwr CCA TAC CGA AAC TCG TTC ACC - Rev CCG CAG ACA TCC TTC AGA ATC - annealing T 56² $;$ abcg1 (NM_009593): Fwr ACT GTT CTG ATC CCC GTA CT - Rev TCT CCA ATC TCG TGC CGT A - annealing $\mathrm{T}^{\circ} 59^{\circ} \mathrm{C}$; apoe (NM_009696): Fwr CAC TCG AGC TGA TCT GTC AC - Rev TGG AGG CTA AGG ACT TGT TTC - annealing $\mathrm{T}^{\circ} 60^{\circ} \mathrm{C}$; scarb1 (NM_016741): Fwr CTT GCT GAG TCC GTT CCA TT - Rev GTA CCT CCC AGA CAT GCT TC - annealing $\mathrm{T}^{\circ} 59^{\circ} \mathrm{C}$; $h p r t$ (NM_013556): Fwr AGC AGG TCA GCA AAG AAC T - Rev CCT CAT GGA CTG ATT ATG GAC A - annealing $\mathrm{T}^{\circ}$ 57 ${ }^{\circ} \mathrm{C}$; rplpo (NM_007475.5): Fwr ATC ACA GAG CAG GCC CTG CA - Rev CAC CGA GGC AAC AGT TGG GT - annealing $\mathrm{T}^{\circ} 57^{\circ} \mathrm{C}$ ). Quantitative PCR were performed using a Rotor-Gene 6000 series (QIAGEN, Toronto, ON, Canada) and data acquired/analysed with the on-board Rotor-Gene series Software version 1.7. All qPCR efficiencies were between $90-110 \%$, with $\mathrm{R}^{2}$ values ranging between $0.97-1.00$. The thermo-protocol was as follows: $95^{\circ} \mathrm{C}$ for $3 \mathrm{~min}$, followed by 40 cycles of $95^{\circ} \mathrm{C}$ for $10 \mathrm{~s}$ and $57-60^{\circ} \mathrm{C}$ for $30 \mathrm{~s}$ and followed by a melting curve. 


\section{Lung function assessment}

Lung functions were assessed using the FlexiVent ${ }^{\circ}$ (SCIREQ, Montreal, PQ, Canada). Mice were anaesthetised with $100 \mathrm{mg} \cdot \mathrm{kg}^{-1}$ ketamine and $10 \mathrm{mg} \cdot \mathrm{kg}^{-1}$ xylazine; tracheotomised with an 18-gauge blunted needle, mechanically ventilated at a respiratory rate of 150 breaths $\cdot \mathrm{min}^{-1}$ and a tidal volume of $10 \mathrm{~mL} \cdot \mathrm{kg}^{-1}$, with a pressure limit of $30 \mathrm{cmH}_{2} \mathrm{O}$. Muscle paralysis was achieved using pancuronium ( $2 \mathrm{mg} \cdot \mathrm{kg}^{-1}$, Sandoz, Boucherville, PQ, Canada) to prevent respiratory efforts during the measurement. The following sequence of measures was repeated three times: Deep inflation, Snapshot-150, Quick Prime-3 and Pressure/Volume-loop to obtain lung inspiratory capacity, resistance, hysteresis, compliance and elastance.

\section{Body composition analyses}

Mice were anaesthetised with isoflurane. Body composition was assessed using the Lunar PIXImus dual-energy X-ray absorptiometry (DEXA) system.

\section{In vitro cholesterol efflux assay}

J774A.1 macrophages (ATCC TIB-67) were grown to 75\% confluence (DMEM, 10\% FBS, 1\% penicillin/ streptomycin, $1 \% \mathrm{~L}$-glutamine) in 48 -well plates. They were then loaded with ${ }^{3} \mathrm{H}$-cholesterol overnight $(0.5 \mu \mathrm{Ci}$ per well; PerkinElmer, Waltham, MA, USA) in DMEM with $1 \%$ FBS. On the next day, cells were thoroughly washed with warm PBS and exposed for $4 \mathrm{~h}$ to polyethylene glycol-treated human or mouse serum $(2.8 \% \mathrm{v} / \mathrm{v}$ final $)$ or 10 -fold concentrated mouse bronchoalveolar lavage $(20 \% \mathrm{v} / \mathrm{v}$ final $)$. Supernatant was collected and macrophages were lysed. Total ${ }^{3} \mathrm{H}$-cholesterol was assessed in both supernatant and macrophage lysate. Percentage of cholesterol efflux was calculated as follow: supernatant/(supernatant + lysate $) \times 100$. In the context of this study, this assay gives information on how the capacity of BAL fluid or serum lipoprotein acceptors to accept cholesterol is affected by cigarette smoke exposure. Please refer to Low et al. [14] for a complete description of the technique and data interpretation.

\section{Statistical analysis}

Two-group comparisons were performed using unpaired student t-tests. Multiple group comparisons were performed using one-way ANOVA followed, if applicable $(\mathrm{p}<0.05)$, by a post hoc Tukey's multiple comparisons test. Tests were performed using Prism 7 from GraphPad Software, Inc.

\section{Results \\ Impact of cigarette exposure on key genes involved in the regulation of reverse lipid transport} To investigate whether cigarette smoke exposure impacts the expression of important genes involved in regulating reverse lipid transport, we compared the expression levels of abca1, abcg1, scarbl and apoe in clinical lung samples from individuals with different smoking status and history [13]. We found that $A B C G 1, S C A R B 1$ and $A P O E$ expressions were higher in active smokers compared to never-smokers, whereas $A B C A 1$ expression was significantly lower (figure 1a). Smoking-induced alterations in abcg1, abcal, scarb1 and apoe lung expression persisted in ex-smokers and progressively reached the levels of never-smokers with increased duration of smoking cessation (figure 1a). With regard to the mouse model of cigarette smoke exposure, abcg1 expression was rapidly increased in BALB/c mice following 2 weeks of exposure whereas abcal and apoe expression was only increased following 24 weeks of exposure (figure 1b). Apoe and $a b c g 1$ levels remained elevated following cessation while abca1 levels returned to baseline (figure $1 \mathrm{~b}$ ). Levels of scarb1 were reduced from 8 weeks of exposure to cigarette smoke and remained lower despite cessation (figure 1b). Altogether, these results show that abcg1, abca1, scarb1 and apoe, central genes involves in reverse lipid transport, are affected by cigarette smoke exposure in both humans and mice.

\section{Impact of cigarette smoke exposure on pulmonary and circulating lipid export capacity}

Since cigarette smoke exposure affects the expression of key genes involved in reverse lipid transport, we investigated the ability of the pulmonary extracellular milieu and peripheral circulation to promote reverse lipid transport. Using an in vitro cholesterol efflux assay, we found that BAL fluid and serum samples collected from BALB/c mice $2 \mathrm{~h}$ after a single $2 \mathrm{~h}$-exposure to cigarette smoke had increased ability to promote reverse lipid transport compared to room air-exposed controls (time point A; figure 2). This phenomenon was also observed in BAL and serum samples collected $2 \mathrm{~h}$ after the last exposure of a 4-day protocol (time point B; figure 2). Interestingly, only BAL fluid samples collected $21 \mathrm{~h}$ after the last cigarette smoke exposure of a 4-day protocol showed higher reverse lipid transport stimulation capacity, whereas no differences were observed between serum samples from room air and cigarette smoke-exposed mice (time point C; figure 2). These data show that, in response to cigarette smoke exposure, there is a rapid biological drive to increase local and peripheral reverse lipid export capacity. 

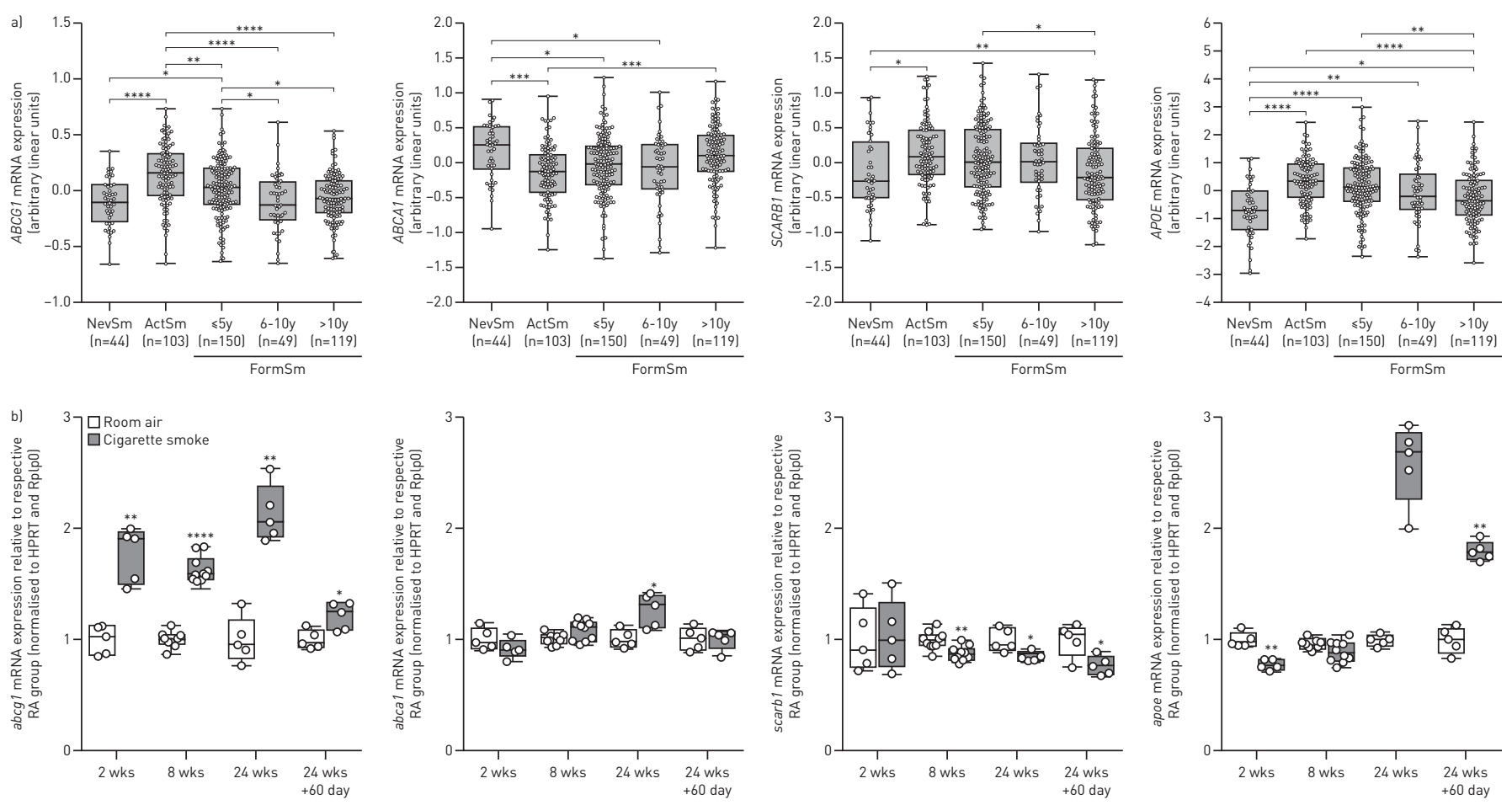

FIGURE 1 Cigarette smoking affects pulmonary expression levels of key genes involved in reverse lipid transport in both humans and mice. a) Pulmonary mRNA expression (microarray) of ABCG1, ABCA1, SCARB1, and APOE in human never smokers (NevSm), active smokers (ActSm), former smokers (FormSm) who stopped smoking for 5 years or less ( $\leqslant 5 \mathrm{y}$ ), 6 to 10 years ago ( $6-10$ y) or over 10 years ago (>10 y). b) Pulmonary mRNA expression (qPCR) of abcg1, abca1, scarb1 and apoe in control room air-exposed BALB/c mice and BALB/c mice exposed to mainstream cigarette smoke for 2 weeks ( 2 wks), 8 weeks ( 8 wks), 24 weeks (24 wks), or 24 weeks followed by a 60-day cessation period (24 wks+60 day). *: $p<0.05 ;{ }^{* *}: p<0.01 ;{ }^{* * *}: p<0.001 ;{ }^{* * *}: p<0.0001$.

ApoA-1 deficiency exacerbates pulmonary and systemic responses to cigarette smoke exposure ApoA-1-deficient mice show a marked reduction in reverse lipid transport capacity as measured using the cholesterol efflux assay (figure 3a). To investigate the impact of dysfunctional reverse lipid transport on the pulmonary and systemic response to cigarette smoke exposure, wildtype C57BL/6J and ApoA-1-deficient

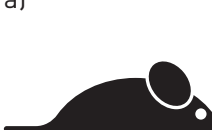

Timepoint

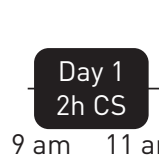

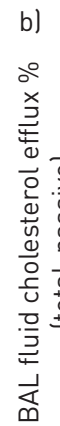

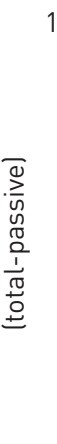

BAL fluid

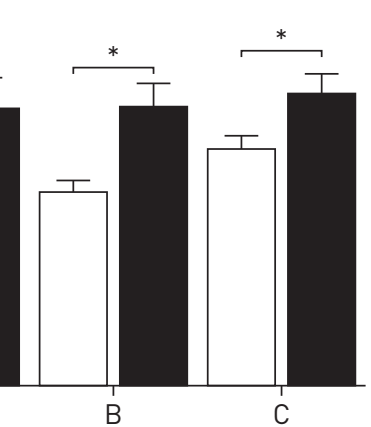

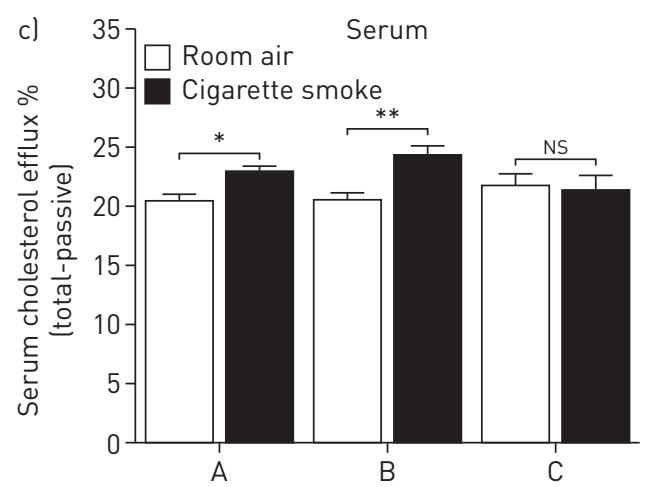

FIGURE 2 Impact of cigarette smoke exposure on pulmonary and systemic reverse lipid export capacity. a) Female 6-8 weeks old BALB/c mice ( $n=5$ per group) were exposed to room air or mainstream cigarette smoke (CS) and sacrificed at three different time points for bronchoalveolar lavage (BAL) and serum collection. Cholesterol efflux assay was performed using b) BAL fluid and c) serum samples collected at time point $A, B$ and $C$. NS: nonsignificant. ${ }^{*}: p<0.05 ;{ }^{* *}: p<0.01$. 

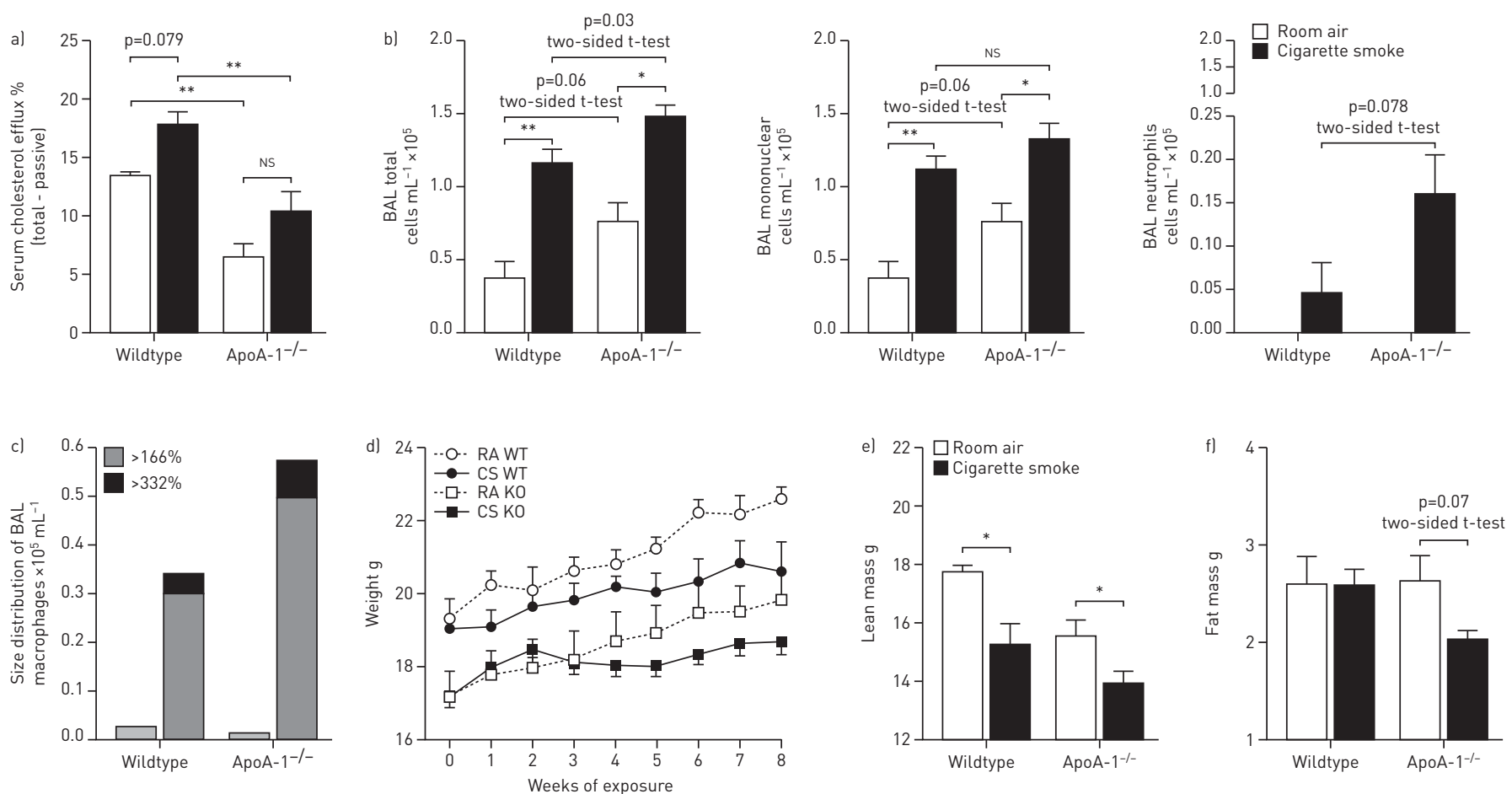

FIGURE 3 Deficiency in ApoA-1 exacerbates the response to cigarette smoke. C57BL/6J and ApoA-1-deficient (C57BL/6J background) mice ( $\mathrm{n}=4-$ 5 per groupl were exposed to room air (RA) or mainstream cigarette smoke (CS) for 8 weeks prior to sacrifice the day after the last exposure. a) Cholesterol efflux assay was performed on serum collected at sacrifice. b) Bronchoalveolar lavage (BAL) total cell counts, cell differentials (mononuclear cells and neutrophils), and c) macrophage size measurements were performed (the 166\% value represents the 95 th percentile and $332 \%$ the 95th percentile $\times 2$ ). d) Mice were weighed on a weekly basis (Monday; weeks 0-7) and the day prior to sacrifice (week 8), along with body composition assessment by DEXA densitometry (e, f). NS: nonsignificant. *: $p<0.05$; ${ }^{* *}: p<0.01$.

mice were exposed to mainstream cigarette smoke for 8 weeks. We found that ApoA-1 deficiency led to an increase in cigarette smoke-induced BAL total cell and neutrophil numbers compared to wildtype animals (figure 3b). Despite similar numbers in mononuclear cells between ApoA-1-deficient and wildtype mice exposed to cigarette smoke, a greater proportion of pulmonary macrophages from ApoA-1-deficient mice were considered enlarged (figure 3c). ApoA-1-deficient mice also had lower pulmonary levels of $a b c a 1$, but smoking-induced changes in abcal, abcg1, scarb1 and apoe were not different between C57BL/6J and ApoA-1-deficient mice (figure S1). With regard to extrapulmonary aspects, Apoal-deficient mice remain significantly smaller than wildtype mice and cigarette smoke exposure inhibited weight gain similarly in both strains (figure $3 \mathrm{~d}$ ). In wildtype mice, cigarette smoke exposure prevents lean mass gain, while adiposity remains similar (figure $3 \mathrm{e}-\mathrm{f}$ ). However, ApoA-1-deficient mice gain significantly less lean and fat mass (figure $3 \mathrm{e}-\mathrm{f}$ ). Altogether, these data show that a reduced capacity to support reverse lipid export can exacerbate pulmonary, and some systemic, effects of cigarette smoke exposure.

\section{Impact of MDCO-216 therapy on the pulmonary response to cigarette smoke}

As ApoA-1-deficient mice appear to be more susceptible to cigarette smoke exposure, we investigated whether boosting lipid export could dampen the pulmonary and systemic effects of cigarette smoke exposure. To do so, we used a recombinant ApoA-1 Milano/phospholipid complex (MDCO-216), which has the ability to promote reverse lipid transport [15], in conjunction to our cigarette smoke exposure models. We found that MDCO-216 injected intraperitoneally rapidly reaches the lungs (figure 4a) and that cigarette smoke exposure does not interfere with its translocation to the pulmonary environment (figure $4 \mathrm{~b}$ ). In the 2-week protocol, cigarette smoke exposure lead to a significant increase in BAL total cellularity and mononuclear cells, which was not observed in MDCO-216-treated animals (20 and $100 \mathrm{mg} \cdot \mathrm{kg}^{-1}$ ) (figure 4c). However, cigarette smoke-induced neutrophilia was not impacted by MDCO-216 treatment (figure 4c). In the 2-week protocol, MDCO-216 significantly reduced BAL pulmonary macrophage size compared to vehicle-treated animals (figure 4d). With regard to the 8-week cigarette smoke exposure protocol, the therapeutic treatment with MDCO-216 had no significant impact on BAL total cell, mononuclear cell, and neutrophil numbers compared to the vehicle treatment, and BAL pulmonary macrophage size remained similar. MDCO-216 therapeutic delivery increased abcal and reduced scarbl 

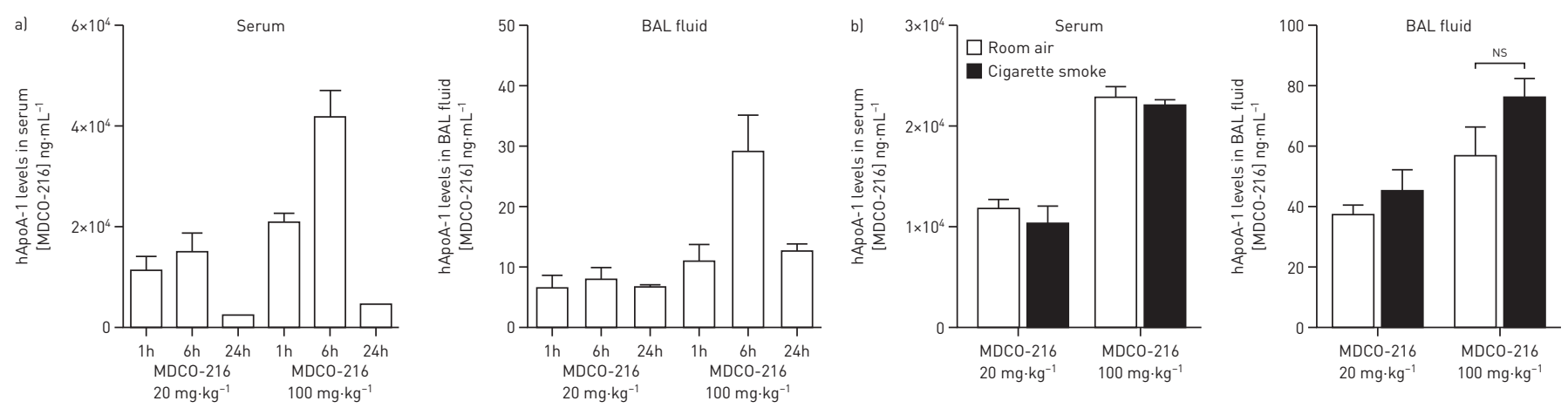

2-week cigarette smoke exposure
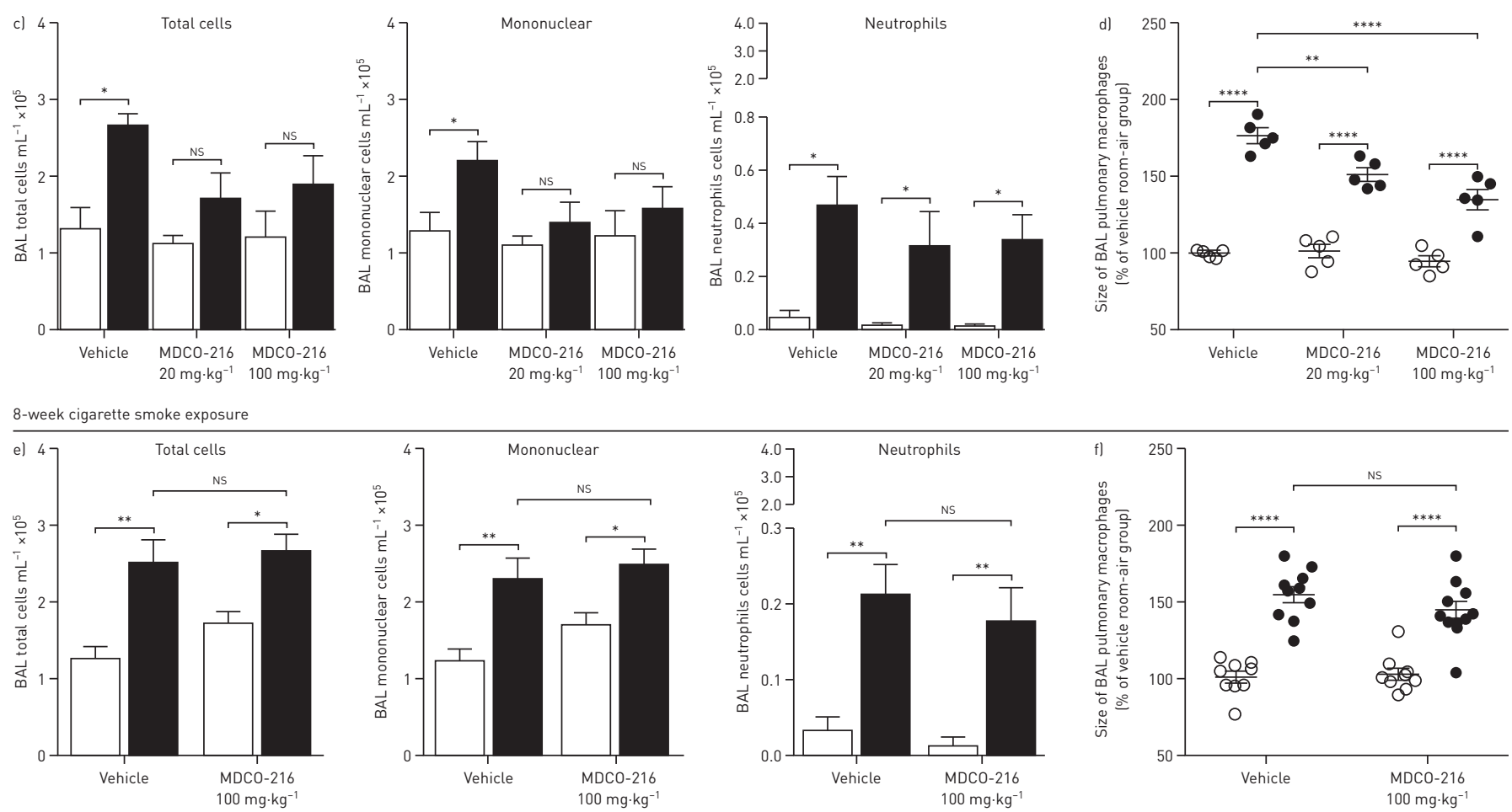

FIGURE 4 Impacts of prophylactic and therapeutic MDCO-216 treatment on the pulmonary response to cigarette smoke. a) Human ApoA-1 levels (surrogate for MDC0-216) were measured in the bronchoalveolar lavage (BAL) and serum of C57BL/6 mice ( $=5$ per group) sacrificed 1, 6, and $24 \mathrm{~h}$ after a single intraperitoneal injection of 20 and $100 \mathrm{mg} \cdot \mathrm{kg}^{-1}$ of $\mathrm{MDCO}-216$. BALB/c mice ( $=4-5$ per group) were exposed to room air or mainstream cigarette smoke for 2 weeks, injected with vehicle, 20 or $100 \mathrm{mg} \cdot \mathrm{kg}^{-1}$ of MDCO-216 on weekdays and sacrificed $18 \mathrm{~h}$ after the last exposure. b) Human ApoA1 levels were measured in the BAL fluid and serum, and c) BAL total cell numbers, differentials and d) macrophage size measurements were performed. BALB/c mice ( $n=9-10$ per group) were exposed to room air or mainstream cigarette smoke for 8 weeks, injected with vehicle or $100 \mathrm{mg} \cdot \mathrm{kg}^{-1}$ of MDCO-216 every second day of weeks $5-8$, and sacrificed $18 \mathrm{~h}$ after the last exposure. e) BAL total cell numbers, differentials and f) macrophage size measurements were performed. NS: nonsignificant. ${ }^{*}: p<0.05 ;{ }^{* *}: p<0.01 ;{ }^{* * * *}: p<0.0001$.

pulmonary mRNA levels, but smoking-induced changes in abcal, abcgl, scarbl and apoe were not different between vehicle and MDCO-216-treated mice (figure S1). These findings are mitigated by the fact that both room air and cigarette smoke-exposed mice that received MDCO-216 started producing antibodies against the compound (human ApoA-1), likely interfering with its biological activity (figure S2). Altogether, these results show that systemic administration of a recombinant ApoA-1 Milano/phospholipid complex (MDCO-216) can reach the lungs and has the potential to dampen mononuclear cells/ macrophages recruitment in the lungs as well as limit their size.

\section{Impact of MDCO-216 therapy on lung functional alterations caused by cigarette smoke}

To investigate the impact of MDCO-216 administration on lung functional alterations caused by cigarette smoke exposure, we performed FlexiVent analyses in mice exposed to cigarette smoke or room air for 8 weeks and treated with either vehicle or MDCO-216 for the last 4 weeks. As expected, cigarette smoke exposure led to significant alterations in the general pressure-volume loop dynamics (figure 5a) and 

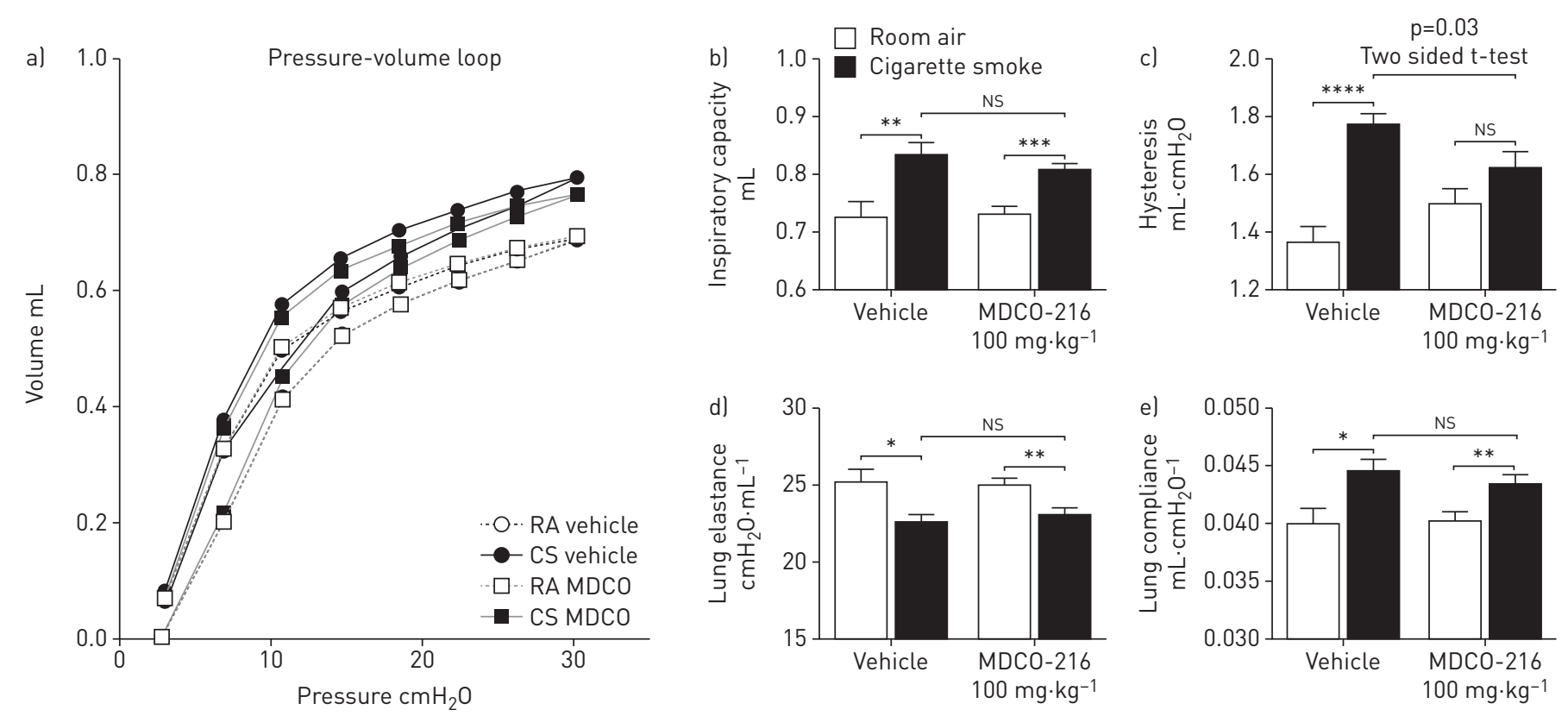

FIGURE 5 Therapeutic MDC0-216 treatment reduces aspects of cigarette smoke-induced lung function alterations. BALB/c mice ( $\mathrm{n}=9-10$ per groupl were exposed to room air (RA) or mainstream cigarette smoke (CS) for 8 weeks, injected with vehicle or $100 \mathrm{mg} \cdot \mathrm{kg}^{-1}$ of MDCO-216 every second day of weeks 5-8, and sacrificed $18 \mathrm{~h}$ after the last exposure. Lung mechanical properties were then assessed using FlexiVent. a) Average pressure-volume loops for each group. b) Inspiratory capacity. c) Hysteresis. d) Lung elastance. e) Lung compliance. NS: nonsignificant. ${ }^{*}: p<0.05 ;{ }^{* *}: p<0.01 ;{ }^{* * *}: p<0.001 ;{ }^{* * * *}: p<0.0001$.

increased lung inspiratory capacity, hysteresis and compliance while reducing lung elastance (figure 5b-e). MDCO-216 treatment only significantly reduced the impact of cigarette smoke exposure on lung hysteresis. These results show that MDCO-216 can have partial effects on lung functional alterations caused by cigarette smoke exposure.

Impact of MDCO-216 therapy on body composition alterations caused by cigarette smoke

To investigate the impact of MDCO-216 treatment on body composition alterations caused by cigarette smoke exposure, we performed whole-body DEXA densitometry analyses before the initiation of the treatments (week 5) and the day before sacrifice (week 8). Vehicle and MDCO-treated mice exposed to cigarette smoke were lighter after 8 weeks of exposure than their room air-exposed counter parts (figure 6a). During the treatment phase, vehicle-treated mice exposed to room air significantly gained more weight than mice exposed to cigarette smoke (figure 6b), largely explained by a gain of fat mass (figure 6c) rather than lean mass (figure 6d). MDCO-216-treated room air-exposed mice show a profile very similar to vehicle-treated room air-exposed mice, MDCO-216 alone having no significant independent effect on body composition. Interestingly, treatment with MDCO-216 induced a marked gain in lean mass in cigarette smoke-exposed mice (figure 6d). These data show that MDCO-216 treatment can have positive effects on body composition of mice exposed to cigarette smoke exposure, notably by increasing lean mass.

\section{Discussion}

Results from several studies suggest that cigarette smoke exposure affects pulmonary lipid homeostasis, notably by directly damaging pulmonary lipids, reducing pulmonary surfactant levels, increasing levels of anti-oxidised lipid antibodies, and causing lipid accumulation in pulmonary macrophages [8, 9, 11, 16-18]. Reverse lipid transport is also a crucial function in lung homeostasis, allowing pulmonary macrophages and structural cells to properly export intracellular lipid. Similar to cigarette smoke exposure, deficiencies in ABCA1 and ABCG1, two instrumental lipid transporters involved in reverse lipid transport, cause lipid accumulation in pulmonary macrophages and chronic lung inflammation [5-7]. Therefore, we investigated the impact of cigarette smoke exposure on aspects of reverse lipid transport in human and mouse lungs as well as the impact of poor reverse lipid transport capacity on the pulmonary and systemic response to cigarette smoke exposure in an in vivo mouse model. We also investigated the potential benefits of promoting reverse lipid transport using a recombinant ApoA-1 Milano/phospholipid complex (MDCO-216). 

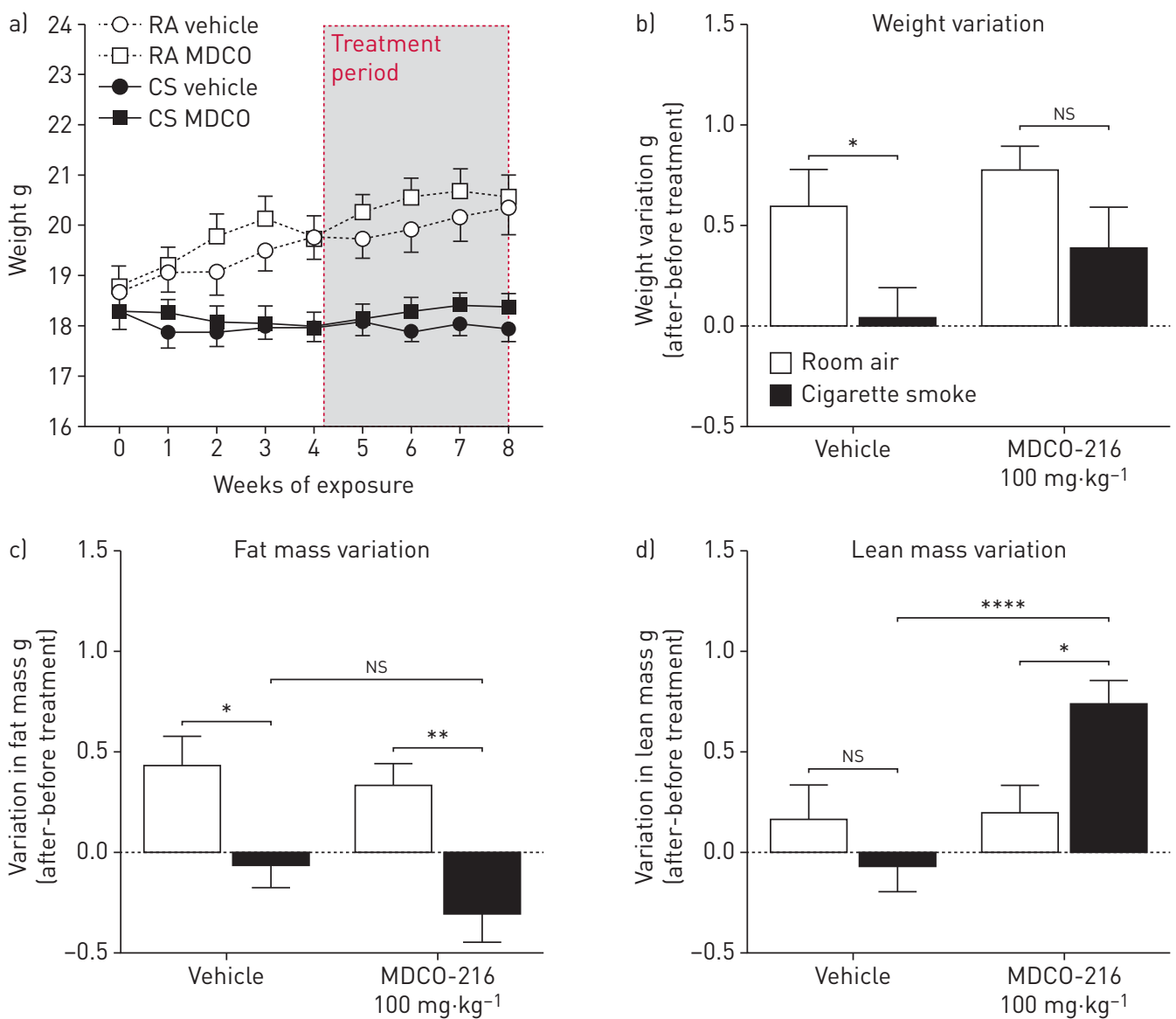

FIGURE 6 Administration of MDCO-216 improves aspects of cigarette smoke-induced changes in body composition. BALB/c mice ( $n=9-10$ per group) were exposed to room air (RA) or mainstream cigarette smoke (CS) for 8 weeks, injected with vehicle or $100 \mathrm{mg} \cdot \mathrm{kg}^{-1}$ of MDCO-216 every second day of weeks $5-8$. Body composition was assessed by DEXA densitometry the day before the initiation of treatments and the day before sacrifice. a) Weekly weight measures. Variations between the end of the protocol and the day the treatments were initiated for mouse b) total weight, c) fat mass and d) lean mass. NS: nonsignificant. ${ }^{*}: p<0.05 ;{ }^{* *}: p<0.01 ;{ }^{* * * *}: p<0.0001$

Smoking modulates pulmonary mRNA levels of abcg1, abcal, scarb1 and apoe in both humans and mice. From the human lung data, we can see that the effects on apoe levels persist for over a decade following cessation, while abcg1, scarb1 and abcal levels take between 5 and 10 years to return to never-smokers' levels. Mouse data show that $a b c g 1$ is rapidly induced upon cigarette smoke exposure, while the effects on abca1, scarb1 and apoe are more progressive in nature. This transcriptional profile associated with smoking in both humans and mice is highly consistent with the notion of increased reverse lipid transport, or at least a biological will to do so. Functionally, ABCA1 exports lipids to lipid-poor HDLs (pre- $\beta$-HDLs) trough ApoA-1 binding while ABCG1 and SR-B1 (protein of the gene scarb1) export lipids to more mature HDLs. Literature is extremely scarce on the use of HDL lipoprotein subsets by the lungs, but our data suggest that, upon cigarette smoke exposure, the lungs activate mechanisms to increase its capacity to export lipids to more mature HDLs through ABCG1 and SR-B1, for instance, pre- $\beta$-HDLs likely being less accessible for ABCA1-mediated processes. While the regulating mechanisms behind $a b c g 1$, abcal, scarb1 and apoe expression in the smoker's lungs remain to be clarified, the expression profile of these four genes crucial to reverse lipid transport supports a strong interaction between smoking and pulmonary reverse lipid transport.

Cigarette smoke exposure rapidly affects reverse lipid transport-stimulating capacity in the mouse lungs and circulation. In fact, an increase in BAL fluid and serum cholesterol efflux-stimulating capacity can be observed as early as $2 \mathrm{~h}$ after a single exposure to cigarette smoke. In humans, reduced reverse lipid transport is observed in individuals with coronary artery disease, for which smoking is an important risk factor. A very limited number of studies, however, looked at the specific effects of smoking in "healthy" young/middle-aged smokers [19]. Smoking is notoriously known to reduce circulating HDL and ApoA-1 
levels, strongly supporting a negative impact on reverse lipid transport. Discrepancies between the effects of smoking on reverse lipid transport may be attributable to differences between species, the HDL/LDL ratio being much lower in humans $(\sim 0.5)$ than in mice $(\sim 2.5)$. Nevertheless, our data suggest that cigarette smoke exposure triggers a local and systemic drive to promote reverse lipid transport capacity in mice. However, this increase in reverse lipid transport-stimulating capability does not appear to be sufficient to prevent pulmonary macrophages from accumulating intracellular lipids.

Mice deficient in ApoA-1 have a reverse lipid transport capacity reduced to about $50 \%$ of wildtype mice and show an exacerbated immune response to cigarette smoke exposure and increased pulmonary macrophage size. Conversely, treatment with MDCO-216 showed potential for reducing the inflammatory response to cigarette smoke, especially mononuclear cell accumulation and pulmonary macrophage size, and aspects of smoke-induced lung function alterations. This displays the significant impact reverse lipid export capacity can have on the magnitude of the pulmonary response to cigarette smoke exposure. Our data is consistent with the study from KIM et al. [20], showing that mice overexpressing human ApoA-1 in the alveolar epithelium are protected from lung emphysema. It also aligns with findings in experimental atherosclerosis, where ApoA-1 deficiency exacerbates the pathology [21, 22], while introduction of a human ApoA-1 transgene confers protection [23, 24]. In both pathologies, the proposed and most likely mechanism of action is promotion/reduction of reverse lipid export. However, HDL molecules and ApoA-1 have been shown to have numerous other functions, including anti-inflammatory and anti-oxidant properties [25]. While each of these mechanisms could have a significant impact on smoking-induced lung inflammation, macrophage lipid accumulation, and lung function alterations, we currently do not have the data to support mechanistic explanations of our findings.

Aside from the pulmonary phenotype, ApoA-1 deficiency and MDCO-216 treatment significantly affected the variations in mouse total weight and body composition attributable to cigarette smoke exposure. ApoA-1 deficient mice are smaller than wildtype mice at baseline, a difference largely attributable to a reduced lean mass, while MDCO-216 did not significantly affect total weight or body composition. The main differences between ApoA-1 deficiency and MDCO-216 treatment in mice exposed to cigarette smoke were that ApoA-1 deficiency caused a significant loss in both lean and fat mass, while MDCO-216 treatment markedly increased lean mass, leaving fat mass unaffected. ApoA-1 is known to affect numerous metabolic functions, including the stimulation of glucose uptake by skeletal muscle [26, 27]. It has also been shown that ApoA-1 can increase mitochondrial biogenesis in vitro in myotubes [28]. While these findings are consistent with an increase in lean mass, we currently cannot explain why this phenotype is only observed in mice exposed to cigarette smoke. It is likely that MDCO-216 favours a regain of the lost lean mass caused by cigarette smoke exposure; potential mechanisms being improved glucose uptake and myogenesis. Additional research is needed to understand the cellular and molecular mechanisms. Clinically, reduced lean/muscle mass has significant adverse effects on quality of life and mortality rate in patients with COPD. Moreover, only a proportion of individuals with COPD show significant limb muscles functional alterations. Reverse lipid transport capacity may represent a novel avenue of investigation to explain the variability in limb muscle phenotypes in smokers with and without COPD and restore limb muscle functionality.

A limitation of this study, inherent to the use of human protein in mice, is the apparition of antibodies against MDCO-216. This very likely reduced the efficacy of the MDCO-216 treatment in the last days or weeks of the protocol, likely having more pronounced impacts in the 8 -week protocol and potentially explaining the reduced impact of MDCO-216 in this setting.

Overall, this study shows that smoking affects mechanisms of pulmonary reverse lipid transport and also how the biological capacity to promote reverse lipid transport impacts the intensity of the pulmonary response to cigarette smoke. This tight interaction between smoking and pulmonary reverse lipid transport aligns with the pathological mechanisms of coronary artery disease and dyslipidaemia, for which smoking is a major risk factor. Research using a more comprehensive approach is required to investigate smoking-related/associated pathologies as a whole as they tend to coexist in many individuals.

\section{Acknowledgements}

Part of the data presented in this manuscript is from the Lung eQTL consortium. Authors would like to sincerely thank the investigators of the consortium, including Peter Paré, Wim Timens, Dirkje S. Postma, Don D. Sin, Ke Hao, and David Nickle.

\section{References}

1 Favari E, Chroni A, Tietge UJ, et al. Cholesterol efflux and reverse cholesterol transport. Handb Exp Pharmacol 2015; 224: 181-206.

2 Phillips MC. Molecular mechanisms of cellular cholesterol efflux. J Biol Chem 2014; 289: 24020-24029.

3 Carey B, Trapnell BC. The molecular basis of pulmonary alveolar proteinosis. Clin Immunol 2010; 135: 223-235. 
4 Goss V, Hunt AN, Postle AD. Regulation of lung surfactant phospholipid synthesis and metabolism. Biochim Biophys Acta 2013; 1831: 448-458.

5 Baldán A, Gomes AV, Ping P, et al. Loss of ABCG1 results in chronic pulmonary inflammation. J Immunol 2008; 180: 3560-3568.

6 Wojcik AJ, Skaflen MD, Srinivasan S, et al. A critical role for ABCG1 in macrophage inflammation and lung homeostasis. J Immunol 2008; 180: 4273-4282.

7 Bates SR, Tao JQ, Collins HL, et al. Pulmonary abnormalities due to ABCAl deficiency in mice. Am J Physio Lung Cell Mol Physiol 2005; 289: L980-L989.

8 Rahman I, van Schadewijk AA, Crowther AJ, et al. 4-Hydroxy-2-nonenal, a specific lipid peroxidation product, is elevated in lungs of patients with chronic obstructive pulmonary disease. Am J Respir Crit Care Med 2002; 166 : 490-495.

9 Aoshiba K, Koinuma M, Yokohori N, et al. Immunohistochemical evaluation of oxidative stress in murine lungs after cigarette smoke exposure. Inhal Toxicol 2003; 15: 1029-1038.

10 Malhotra D, Thimmulappa R, Navas-Acien A, et al. Decline in NRF2-regulated antioxidants in chronic obstructive pulmonary disease lungs due to loss of its positive regulator, DJ-1. Am J Respir Crit Care Med 2008; 178: 592-604.

11 Morissette MC, Shen P, Thayaparan D, et al. Disruption of pulmonary lipid homeostasis drives cigarette smoke-induced lung inflammation in mice. Eur Respir J 2015; 46: 1451-1460.

12 Ueyama K, Yokode M, Arai H, et al. Cholesterol efflux effect of high density lipoprotein is impaired by whole cigarette smoke extracts through lipid peroxidation. Free Radic Biol Med 1998; 24: 182-190.

13 Bosse Y, Postma DS, Sin DD, et al. Molecular signature of smoking in human lung tissues. Cancer Res 2012; 72 : 3753-3763.

14 Low H, Hoang A, Sviridov D. Cholesterol efflux assay. J Vis Exp 2012; e3810.

15 Kallend DG, Reijers JA, Bellibas SE, et al. A single infusion of MDCO-216 (ApoA-1 Milano/POPC) increases ABCA1-mediated cholesterol efflux and pre-beta $1 \mathrm{HDL}$ in healthy volunteers and patients with stable coronary artery disease. Eur Heart J Cardiovasc Pharmacother 2016; 2: 23-29.

16 Pratt SA, Finley TN, Smith MH, et al. A comparison of alveolar macrophages and pulmonary surfactant(?) obtained from the lungs of human smokers and nonsmokers by endobronchial lavage. Anat Rec 1969; 163: 497-507.

17 Finley TN, Ladman AJ. Low yield of pulmonary surfactant in cigarette smokers. N Engl J Med 1972; 286: 223-227.

18 Thayaparan D, Shen P, Stämpfli MR, et al. Induction of pulmonary antibodies against oxidized lipids in mice exposed to cigarette smoke. Respir Res 2016; 17: 97.

19 Kralova Lesna I, Poledne R, Pagacova L, et al. HDL and apolipoprotein Al concentrations as markers of cholesterol efflux in middle-aged women: interaction with smoking. Neuro Endocrinol Lett 2012; 33: Suppl. 2, $38-42$.

20 Kim C, Lee JM, Park SW, et al. Attenuation of cigarette smoke-induced emphysema in mice by apolipoprotein A-1 overexpression. Am J Respir Cell Mol Biol 2016; 54: 91-102.

21 Moore RE, Kawashiri MA, Kitajima K, et al. Apolipoprotein A-I deficiency results in markedly increased atherosclerosis in mice lacking the LDL receptor. Arterioscler Thromb Vasc Biol 2003; 23: 1914-1920.

22 Moore RE, Navab M, Millar IS, et al. Increased atherosclerosis in mice lacking apolipoprotein A-I attributable to both impaired reverse cholesterol transport and increased inflammation. Circ Res 2005; 97: 763-771.

23 Plump AS, Scott CJ, Breslow JL. Human apolipoprotein A-I gene expression increases high density lipoprotein and suppresses atherosclerosis in the apolipoprotein E-deficient mouse. Proc Natl Acad Sci USA 1994; 91: 9607-9611.

24 Pászty C, Maeda N, Verstuyft J, et al. Apolipoprotein AI transgene corrects apolipoprotein E deficiency-induced atherosclerosis in mice. J Clin Invest 1994; 94: 899-903.

25 Rosenson RS, Brewer HB, Ansell BJ, et al. Dysfunctional HDL and atherosclerotic cardiovascular disease. Nat Rev Cardiol 2016; 13: 48-60.

26 Domingo-Espín J, Lindahl M, Nilsson-Wolanin O, et al. Dual actions of apolipoprotein A-I on glucose-stimulated insulin secretion and insulin-independent peripheral tissue glucose uptake lead to increased heart and skeletal muscle glucose disposal. Diabetes 2016; 65: 1838-1848.

27 Cochran BJ, Ryder WJ, Parmar A, et al. In vivo PET imaging with [(18)F]FDG to explain improved glucose uptake in an apolipoprotein A-I treated mouse model of diabetes. Diabetologia 2016; 59: 1977-1984.

28 Song P, Kwon Y, Yea K, et al. Apolipoprotein al increases mitochondrial biogenesis through AMP-activated protein kinase. Cell Signal 2015; 27: 1873-1881. 